\title{
Structural priming and the phrasal/clausal distinction: The case of concealed questions
}

Gözde Bahadır¹, Maria Polinsky²

${ }^{1}$ Cognitive Science, Middle East Technical University, Turkey

${ }^{2}$ Linguistics, Harvard University, USA

https://doi.org/10.36505/ExLing-2011/04/0003/000172

\begin{abstract}
This paper investigates whether structural priming is sensitive to the phrasal vs. clausal nature of the constructions it tests. To that end, we examine NPs that receive a question-like interpretation when embedded under certain predicates. These NPs are known as "concealed question (CQ) NPs". We first report the results of a pilot study that establishes co-occurrence patterns of the target embedding predicates. We then present two structural priming studies which test CQ NPs with "overt embedded questions": "embedded wh-questions" and "embedded declaratives". Both written sentence completion tasks demonstrate structural priming, which turns out to be sensitive to the phrase-clause distinction.
\end{abstract}

Key words: structural priming, concealed questions, embedded clauses, interrogative, declarative

\section{Introduction}

This paper investigates whether structural priming is sensitive to the phrase-clause distinction by testing priming for concealed questions (CQs).

\section{Structural priming}

Structural priming (Bock 1986) is the facilitating effect of experience with a certain morphosyntactic form on future processing. For example, when participants are asked to describe an event that could be expressed with either a prepositional object (PO) (e.g. 'to a little boy' in (1)) or a direct object (DO) (e.g., 'a little boy' in (2)), they produce more PO utterances after a PO prime (1) than after a DO prime (2), and more DO sentences after a DO prime.

(1) The man gave a new toy to a little boy.

(2) The man gave a little boy a new toy.

The same results hold for the active/passive alternation (Bock, 1986). Priming has also been demonstrated with different methodologies such as written sentence completion tasks (Pickering and Branigan 1998).

\section{Concealed questions}

Certain noun phrases (NPs) in English and other languages encode the meaning of a question without taking the form of one. These are aptly called concealed questions (CQs). In a nutshell, CQs involve shifting the meaning of a functional NP argument (3a) to a question-like meaning (3b)

ExLing 2011: Proceedings of 4th Tutorial and Research Workshop on Experimental Linguistics, 25-27 May, Paris, France 
when that NP appears as the object of certain embedding predicates (Heim 1979, Nathan 2006, a.o.).

(3a) The committee announced [the winner of the award].

(3b) The committee announced [who had won the award].

It has been argued that semantically CQs may not have the same denotation as a plain matrix interrogative (i.e., a set of propositions), but may instead behave more like an embedded declarative that-clause conveying the complete true answer to the question (i.e., a proposition) (Nathan 2006). Hence (4a) may be better paraphrased as a declarative (4c) rather than as an interrogative (4b).

(4a) Kim told me [the capital of France].

(4b) Kim told me [what the capital of France was].

(4c) Kim told me [that Paris is the capital of France].

\section{Experiments}

The goal of our study was twofold: to investigate possible priming with CQs and to use the experimental data to distinguish between the competing analytical approaches to CQs, as shown in (4b) vs. (4c). We report three experiments: a pilot study and two priming studies.

\section{Pilot study}

We conducted an offline cloze test with 24 sentence fragments to examine the natural frequencies of co-occurrence of CQ NPs and overt embedded questions (OECs).

(5) The observer reported

Experimental fragments contained two sets of embedding matrix verbs: explain, learn, find out, figure out, report, disclose (Set 1); predict, estimate, determine, discover, announce, guess (Set 2).

The pilot study $(\mathrm{N}=19)$ provided us with a profile for each individual verb. In terms of frequencies, most completions include that-clauses (38.33\%), but there is also a significant number of NPs (31.67\%). The percentage of embedded wh-questions is the lowest (11.11\%).

\section{Priming study 1: NPs and embedded questions}

In the paper-based comprehension-to-production priming experiment, 44 participants were handed 4-page booklets with 60 items (12 Prime-Target pairs and 36 fillers). They read the prime sentences and completed the matching target fragments. Half of the primes contained CQ NPs (6a), and the other half contained embedded questions (EQs) (7a). Target fragments (6b) and (7b) were to be completed by the participants.

(6a) The jeweler explained the value of the necklace.

(6b) The engineer estimated

(CQ NP-Prime)

(7a) The expert explained what the diamond was worth.

(7b) The technician estimated 
Participants produced more NP completions than EQ completions (significant main effect of Completion Type: $F=31,515 ; p=.00 ; \eta^{2}=.429$ ), consistent with the results of the pilot study.

There was also a significant two-way interaction between Prime Type and Completion Type: $\mathbf{F}=\mathbf{5 , 3 9 8} ; \mathbf{p}<.05 ; \eta^{2}=.114$. Participants produced more NP-responses after NP-primes than after EQ-primes, and more EQ-responses after EQ-primes. Therefore, despite the general high frequency of NPs, there is priming in the alternation between NPs and embedded-questions. Due to frequency, this priming is not symmetrical and seems to work better for NPs than for questions.

\section{Priming study 2: NPs and embedded declaratives}

On a theoretical plane, it is unclear whether CQs correspond to interrogative (4b) or declarative embeddings (4c). To investigate the distribution of clausal primes, the previous priming study was conducted using embedded declaratives (EDs) (8) instead of EQ primes (7a).

(8) The expert explained that the diamond was worth five thousand dollars.

In a web-based adaptation of the same task as in Priming Study $1^{1}, 20$ participants read and completed the sentences.

There is a significant main effect of Prime Type $(F=6,211 ; p<.05$; $\eta^{2}=.257$ ). This means that whether the prime is nominal or clausal has an effect on the completions overall: CQ NPs prime NPs, and EDs prime clausal completions. In addition to the nominal and clausal completions, we also have a third type of completion, which is neither an NP nor an ED, with that:

(9) He remembered ...how to write...

There is a significant interaction between Prime Type and Completion Type $\left(\mathbf{F}=\mathbf{5 , 4 9 2 ;} \mathbf{p}<. \mathbf{0 5} ; \boldsymbol{\eta}^{2}=. \mathbf{2 3 4}\right)$ as in the previous study. This suggests that participants provided more NP completions after NP primes than after ED primes and more ED completions after ED primes than after NP primes. The priming effect is confirmed for the CQ NP-ED pair, as well.

\section{Discussion}

Concealed questions have an effect on structural priming when paired with overt embedded questions, expressed either as embedded interrogatives or embedded declaratives. These results provide novel evidence for structural priming being sensitive to the distinction between phrases and clauses.

Researchers using primary data have proposed that concealed questions could be interpreted as embedded wh-questions or as embedded polar interrogatives. In our study, a large proportion of the ED responses would be more appropriate as an answer to a yes-no-question than to a wh-question (e.g., The police disclosed that the suspect was found.). This suggests that while EDs prime EDs, their denotation may be more general than the one 
that corresponds to the denotation of CQs. Thus, our results underscore the contrast between wh-questions and yes-no-questions and indirectly suggest that the wh-question interpretation may be more appropriate for CQs.

\section{Notes}

1. We also adapted CQ NP-EQ priming study to an internet-based format. As previously, there is a significant main effect of answer type $(F=15,725 ; p<.01$; $\left.\eta^{2}=.466\right)$ with more NP completions than EQ completions. However, here we don't get the significant prime type*completion type interaction. This could be due to the differences in methodology or to the smaller sample size (20 vs. 44).

\section{Acknowledgements}

We acknowledge TÜBİTAK and Harvard University FAS for their support. We are grateful to I. Caponigro, P. Graff, A. Hohenberger, E. Kravtchenko, A. M. Morgan, E. R. Su, and participants of the 6th International Workshop on Language Production in Edinburgh for their valuable help and comments.

\section{References}

Bahadir, G., Polinsky M. 2010. Structural Priming of CQs. Poster presented in the 6th International Workshop on Language Production, Edinburgh, UK.

Bock, J.K. 1986. Syntactic persistence in language production. Cognitive Psychology 18, 355-387.

Caponigro, I., Heller, D. 2007. The non-concealed nature of free relatives: Implications for connectivity in specificational sentences. In Barker, C., Jacobson, P. (eds.), Direct Compositionality, 237-263. Oxford, UK, OUP.

Heim, I. 1979. Concealed Questions. In Bäuerle, R., Egli, U., von Stechow, A. (eds.), Semantics from different point of views, 51-60. Berlin, Springer-Verlag.

Nathan, L. 2006. On the interpretation of CQs. PhD Thesis, MIT, MA.

Pickering, M.J., Branigan, H.P. 1998. The representation of verbs: Evidence from syntactic priming in language production. JML 39, 633-651. 